\title{
Current Status of Pediatric Robot-Assisted Surgery in Italy: Epidemiologic National Survey and Future Directions
}

\author{
Ciro Esposito, MD, PhD, ${ }^{1}$ Lorenzo Masieri, MD, ${ }^{2}$ Marco Castagnetti, MD, ${ }^{3}$ Gloria Pelizzo, MD, ${ }^{4}$ \\ Mario De Gennaro, MD, ${ }^{5}$ Gabriele Lisi, MD, ${ }^{6}$ Giovanni Cobellis, MD, ${ }^{7}$ Piergiorgio Gamba, MD, \\ Vincenzo Di Benedetto, $\mathrm{MD}^{9}$, and Maria Escolino, $\mathrm{MD}^{1}$
}

\begin{abstract}
Background: Pediatric robot-assisted surgery (RAS) is gaining increasing acceptance. We aimed to assess the diffusion of pediatric RAS in Italy, the training period, indications, preliminary outcomes, and limitations.

Materials and Methods: An online questionnaire-based survey was performed. The data about robotic activity of 9 Italian Pediatric Surgery units were collected and analyzed.

Results: Most of the participating centers $(7 / 9,77.8 \%)$ started RAS less than 5 years ago with only 2 centers (22.2\%) performing RAS since 2010. The training included dry-lab in 5/9 centers (55.5\%), wet-lab in 5/9 centers $(55.5 \%)$, and robot simulator in $7 / 9$ centers $(77.8 \%)$, followed by an exam to obtain a certificate. The average duration of training was 23.7 hours (range 5-50). A total of 209 robotic procedures was performed in all centers during the period 2010-2018 and included 119 urological (56.9\%), 31 gynecological (14.8\%), 41 gastrointestinal (19.6\%), 12 oncological $(5.7 \%)$, and 6 other $(2.8 \%)$ procedures. The docking time significantly fell down after 18 robotic procedures $(P=.001)$. Intraoperative complications were recorded in 4 cases $(1.9 \%)$. Conversion to laparoscopy was needed in 4 cases $(1.9 \%)$ whereas conversion to open was required in 6 cases (2.8\%). Postoperative complications occurred in 17/209 cases $(8.1 \%)$ and were graded Clavien I-II in 14 cases (6.7\%) and Clavien IIIb in only 4 cases (1.9\%).

Conclusions: Our study confirmed that RAS has still a limited diffusion in Italy for pediatric patients. Before starting robotic activity, pediatric surgeons have to obtain a certificate after a virtual and experimental training period. A mentorship clinical period of 10 cases under supervision of a proctor is also needed. The main indications in children remain reconstructive urological procedures. RAS is safe in children but its applications are currently limited to patients older than 2 years and with a weight $>15 \mathrm{~kg}$, due to the size of robotic ports.
\end{abstract}

Keywords: robot, children, complications, training, docking, team

\section{Introduction}

$\mathbf{R}$ OBOT-ASSISTED SURGERY (RAS) is becoming a clinical reality in Italy during the last few years. ${ }^{1}$ The main fields of application are urology, general surgery, and gynecology. More recently, novel fields of application of RAS including thoracic surgery, cardiac surgery, otolaryngology, and pediatric surgery, have been discovered with encouraging results. ${ }^{2,3}$

RAS has been mainly adopted to perform reconstructive procedures in children, demonstrating the consistent advantage to overcome the technical challenges of such procedures

\footnotetext{
${ }^{1}$ Pediatric Surgery Unit, Federico II University of Naples, Naples, Italy.

${ }^{2}$ Pediatric Urology Unit, Meyer Children Hospital, Florence, Italy.

${ }^{3}$ Pediatric Urology Unit, Medical University of Padua, Padua, Italy.

${ }_{5}^{4}$ Pediatric Surgery Unit, Children's Hospital G. Di Cristina, ARNAS Civico-Di Cristina-Benfratelli, Palermo, Italy.

${ }^{5}$ Pediatric Urology Unit, Bambin Gesù Children Hospital, Rome, Italy.

${ }^{6}$ Pediatric Surgery Unit, G. D’Annunzio University of Chieti, Pescara, Italy.

${ }^{7}$ Pediatric Surgery Unit, Azienda Ospedaliero-Universitaria, Ancona, Italy.

${ }^{8}$ Pediatric Surgery Unit, Medical University of Padua, Padua, Italy.

${ }^{9}$ Pediatric Surgery Unit, University Hospital of Catania, Catania, Italy.
} 
compared with conventional laparoscopy as robotic arms have $7^{\circ}$ of freedom and can articulate up to $90^{\circ} .4,5$ Other advantages related to the use of EndoWrist technology include three-dimensional (3D) high-definition view of the operative field, and improved ergonomics since the surgeon can operate seated on the robotic console, with alignment of eyes and hands' position with the instruments and elimination of hands' tremor. The use of robot in complex reconstructive procedures has also been associated with a shorter learning curve compared with conventional laparoscopy. ${ }^{6,7}$

Despite all these technical advantages of robot, its largescale application has still several limitations in children., 8 The largest barrier is represented by the significant cost of acquiring and maintaining the da Vinci robot. Another important challenge is to adapt the big robotic platform, originally designed for adults, to the small dimensions of children. Modifications and arrangements of the equipment and positioning of the patient and trocars are required. ${ }^{10}$

Robot-assisted laparoscopic pyeloplasty (RALP) is, to date, the most frequent procedure performed in the pediatric population, and the only one where outcomes have been proved to be at least comparable to the open or laparoscopic techniques. ${ }^{11,12}$ The most important limitation is still represented by the costs, which remain higher than the open approach. ${ }^{8,9}$ Many other applications of RAS, including ureteral reimplantation, appendico-vesicostomy creation, bladder neck reconstruction, and kidney stone surgery, have been described in children but further evidence is needed to validate these indications. ${ }^{8,9,13}$

The aim of this multicentric survey was to give an overview about the diffusion of pediatric RAS in Italy, to show how the training period is structured in Italy and to assess the main indications and the preliminary outcomes of RAS in pediatric patients. Additionally, we aimed to outline the main limitations for a widespread diffusion of RAS in pediatric patients.

\section{Materials and Methods}

A questionnaire-based survey was sent via e-mail to all Pediatric Surgery units in Italy and the answers were collected and shared across the participants through SurveyMonkey, an online survey development software. Nine Pediatric Surgery units performing RAS participated in the survey and the data about the robotic activity of all these centers were collected and analyzed.

We assessed the duration of robotic activity and the number of surgeons performing RAS in each center. We also evaluated the modality of training performed by each participating surgeon before starting RAS in vivo. Finally, we assessed volume, type, and outcome of RAS in each participating center. Outcome parameters measured were docking time, intra- and postoperative complications, re-operations, conversions to laparoscopy, and conversions to open surgery. Postoperative complications were graded according to Clavien-Dindo classification system. ${ }^{14}$

The study received the appropriate Institute Review Board (IRB) approval at each participating center.

\section{Results}

Most of the participating centers $(7 / 9,77.8 \%)$ started the robotic activity less than 5 years ago with only 2 centers
(22.2\%) performing RAS since 2010 . The average number of surgeons performing RAS was 2 for each center. All participating surgeons practiced a high-volume laparoscopic activity (>500 laparoscopic procedures/year) before moving to robotics.

In each participating center, the robotic surgeons had to pass an exam after a period of virtual and practical training to obtain a certificate that allowed them to operate "legally covered" using Intuitive robotic system. The robotic training included dry-lab sessions on pelvic trainer in $5 / 9$ centers $(55.5 \%)$, wetlab sessions on animal model in $5 / 9$ centers $(55.5 \%)$, and training on robot simulator in $7 / 9$ centers $(77.8 \%)$ (Fig. 1$)$. The average duration of training before starting RAS in vivo was 23.7 hours (range 5-50).

During the clinical training period, the beginner robotic surgeons at each participating center needed a mentorship period of 10 cases with supervision of an expert robotic surgeon, called "proctor," who was seated on the second robotic console to help the main surgeon in case of technical challenges. In addition, the presence of a robotic technician in the operative room was required for the first 6-12 months of the robotic activity to help in case of problems with the robot's setting.

Regarding the robotic activity, 7/9 participating centers (77.8\%) adopted $\mathrm{Xi}$ model of the da Vinci robot whereas only 2 centers $(22.2 \%)$ adopted Si model. A total of 209 robotassisted procedures was performed in all centers during the period 2010-2018. They included 119 urological procedures (56.9\%), 31 gynecological procedures (14.8\%), 41 gastrointestinal procedures $(19.6 \%), 12$ oncological procedures (5.7\%), and 6 other procedures (2.8\%) (Fig. 2). A laparoscopic assistant port was always adopted in $7 / 9$ centers $(77.8 \%)$ whereas it was adopted in selected cases (urological procedures, upper abdominal surgery) in only 2 centers $(22.2 \%)$. The docking time significantly fell down after 18 robotic procedures $(P=.001)$ (Fig. 3).

Intra-operative complications occurred in 4 cases $(1.9 \%)$ and included minimal blood loss or spillage of ovarian dermoid cyst, not requiring transfusion or further therapeutic measures. Conversion to laparoscopic surgery was needed in 4 cases $(1.9 \%)$ whereas conversion to open surgery was required in 6 cases $(2.8 \%)$. Postoperative complications rate occurred in $17 / 209$ cases $(8.1 \%)$ and were graded Clavien I-II in 14 cases $(6.7 \%)$ and Clavien IIIb in only 4 cases $(1.9 \%)$. Re-operations rate was $1.9 \%$ and included removal of a malfunctioning ureteral stent via cystoscopy in 3 patients who underwent Anderson-Hynes (AH) pyeloplasty and laparoscopic exploration with drainage of uroperitoneum in 1 patient who underwent AH pyeloplasty.

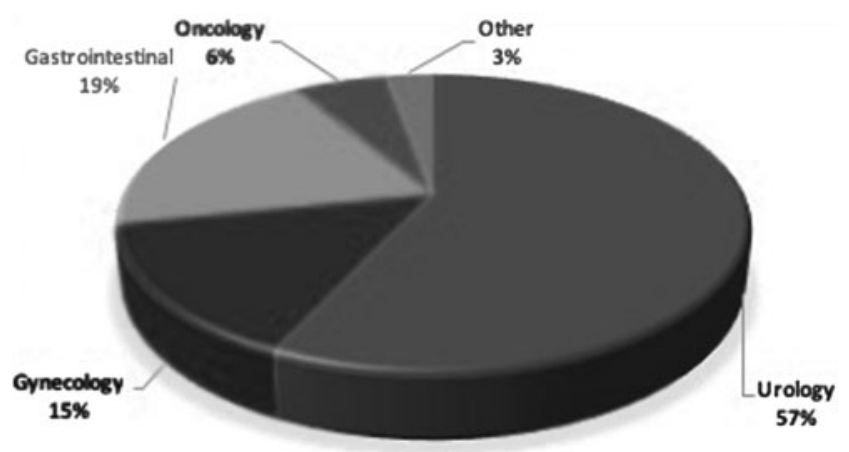

FIG. 1. Training before RAS. RAS, robot-assisted surgery. 


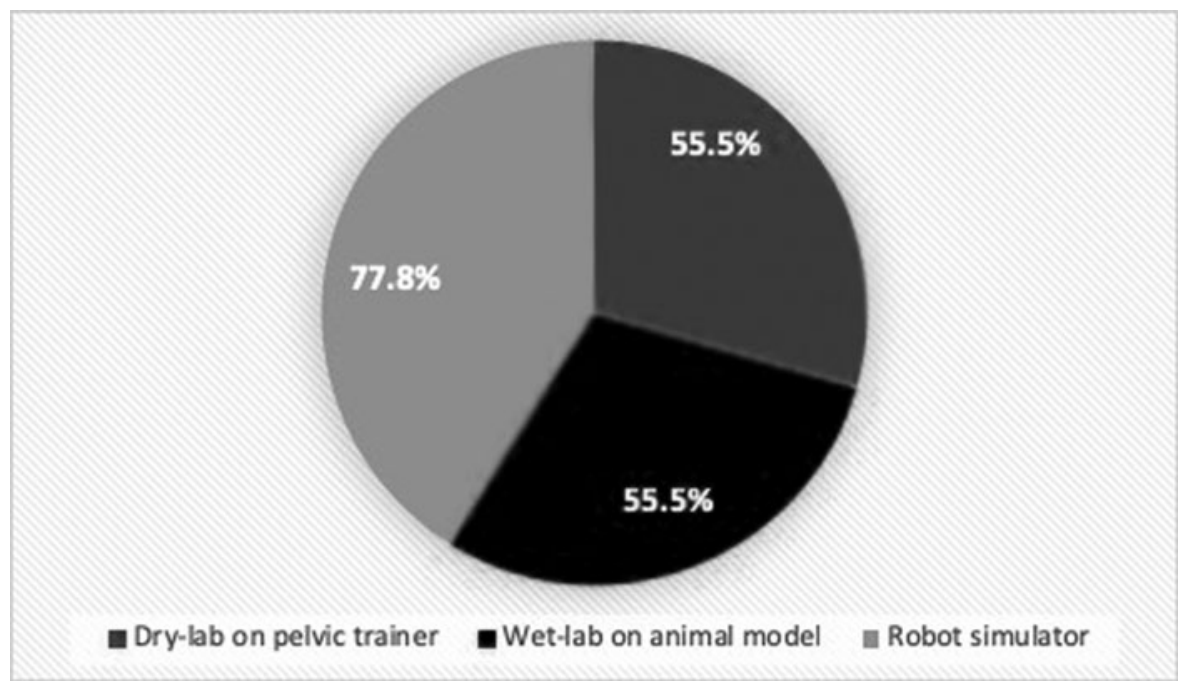

FIG. 2. Principal indications for pediatric RAS in Italian centers. RAS, robot-assisted surgery.

\section{Discussion}

Minimally invasive surgery (MIS) has gained a widespread diffusion for surgical treatment of different pathologies in children with reported advantages of decreased postoperative pain and hospitalization time, a quick postoperative recovery, and better cosmetic results. ${ }^{15}$ The introduction of robotic platform represented a further step in the evolution of MIS. The advantages of robot technology include 3D highdefinition view of the operative field, comfortable surgeon's ergonomics, and improved instruments' dexterity in complex reconstructive procedures. ${ }^{16,17}$ The main fields of application are urology, general surgery, and gynecology, in which RAS demonstrated several advantages compared to traditional approaches. ${ }^{1}$ However, most of the available evidence is only focused on the adult population.

Reconstructive procedures are more often performed in children compared with adults, in whom most of surgery is demolitive. Additionally, the child has peculiar characteristics such as smaller working space and more delicate tissue han- dling that add further challenges to surgery. ${ }^{10}$ Furthermore, intracorporeal suturing and knotting using the available laparoscopic instruments requires advanced technical skills with use of very small suture sizes and is time consuming. The robot, instead, may help to overcome all these challenges. Several studies have demonstrated that use of robot may shorten the learning curve of intracorporeal suturing, as robotic arms have $7^{\circ}$ of freedom and can articulate up to $90^{\circ}$.,5,18

Thanks to all these advantages, RAS is gaining a widespread diffusion in pediatric MIS. Pediatric urology is one of the most common and useful fields of application of robotic technology, as confirmed in different studies. ${ }^{9,13,19}$ RALP is currently the most frequent procedure performed in the pediatric population, and the only one where outcomes have been proved to be at least comparable to the open or laparoscopic techniques. ${ }^{11,12,20}$ Many other applications of RAS, including ureteral reimplantation, appendico-vesicostomy creation, bladder neck reconstruction, and kidney stone surgery, have been described in children but further evidence is needed to validate these indications. ${ }^{8,9,13,21}$

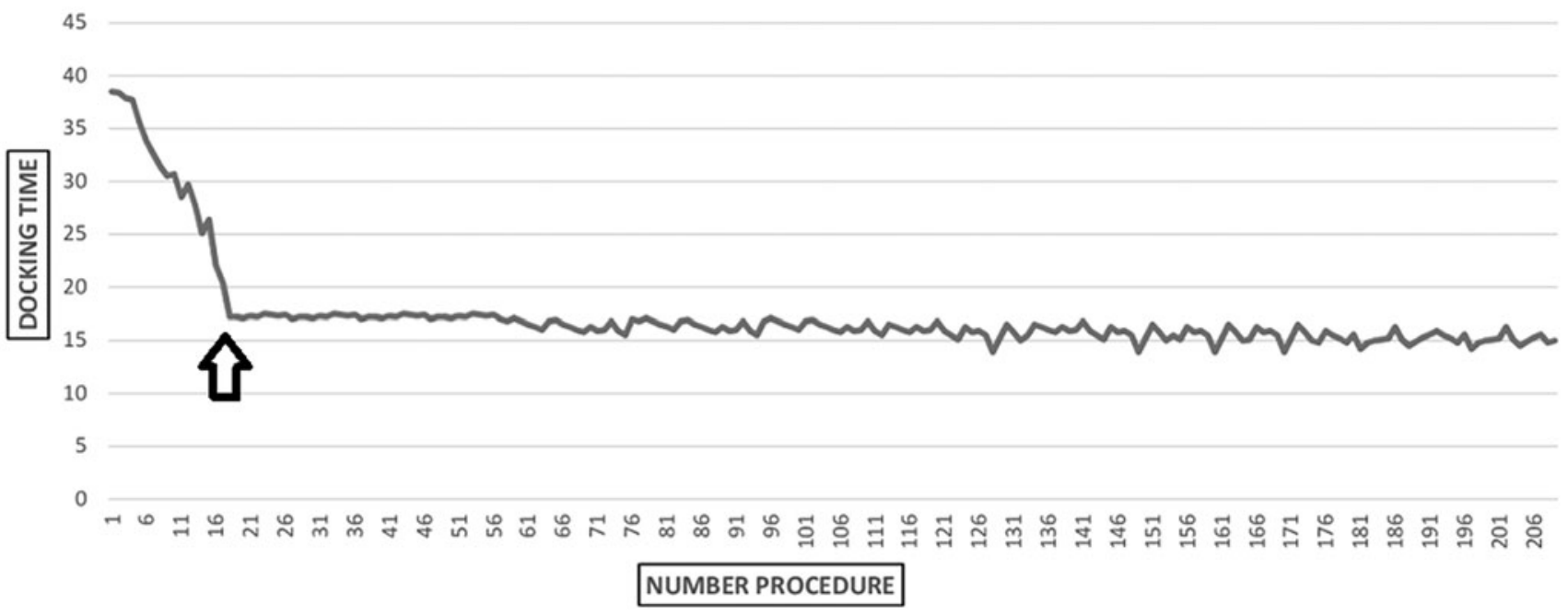

FIG. 3. Learning curve for docking time. 
Despite all the reported advantages, there are still several limitations to a large-scale application of RAS in pediatric population, as demonstrated by lack of large case series and comparative studies between RAS and traditional approaches. The first limitation is represented by low-weight children and infants. We believe that application of RAS is best suited for children with a body weight $>15 \mathrm{~kg}$ to have enough space on the abdominal wall to place three $8-\mathrm{mm}$ robotic ports plus the additional 5-mm laparoscopic assistant port and avoid the clashing between the ports.

Since the DaVinci robot was originally designed for exclusive use in adults, its application in young children is possible only if adaptations and modifications of patient's and ports' position and robot docking are performed. ${ }^{10,22}$ In fact, the robotic ports are generally positioned in the same line to perform several adult procedures, whereas in children we often need to triangulate the robotic ports' position, to achieve a better ergonomics and avoid external conflicts between the robotic arms. We usually adopt an $8 \mathrm{~cm}$ distance between the robotic ports and a 5-7 cm distance between the robotic ports and the laparoscopic assistant port.

Successful application of RAS in infants should take into consideration all technical peculiarities of this patient population. First, they are particularly sensible to the effects of pneumoperitoneum. ${ }^{23,24}$ In infants, this surgery should be safely accomplished limiting the insufflation time and keeping the insufflation pressure as low as possible. ${ }^{10}$

Additionally, a correct setup of the operating room, including positioning of the robot, robotic console, scrub table, and anesthesia machines, is crucial to guarantee an optimal collaboration and communication among all components of the robotic team (main surgeon, assistant surgeon, anesthesiologists, and nurses) during surgery and preserve the patient's safety. ${ }^{25}$ With Si robot system, it is recommended to limit the robot's manipulation by maintaining the robot at one fixed position in the operating room and then moving/rotating the operative table into the final position. ${ }^{10}$ With Xi robot system, the robotic arms can be rotated to the selected position without changing the position of the operative table. ${ }^{10}$

Our study outlined the importance of adequate training before starting the robotic activity. A previous laparoscopic experience was very useful to shorten the learning curve, as demonstrated in our study that reported a short duration of training (23.7 hours) before starting RAS in vivo. In Italy robotic credentials are required to do robotic surgery. In each participating center, the robotic surgeons had to pass an exam after a period of virtual and practical training to obtain a certificate that allowed them to operate "legally covered" using Intuitive robotic system. Furthermore, we believe that this training is crucial not only for the main surgeon but also for the bedside surgeon and the entire robotic team to shorten the docking time. RAS is a team work and requires a welltrained nursing team. ${ }^{10}$

Since robotic procedures are rarely performed in infants, standardized protocols and check-lists can be very useful tools to minimize confusion and practice variations. ${ }^{26-28}$ In our opinion, it is crucial to perform RAS at least once a week to maintain regular the learning curve. ${ }^{29,30}$

Another important consideration emerging from this study was the importance to perform the first robotic procedures with the assistance of a proctor. During the clinical training period, the beginner robotic surgeons at each participating center needed a mentorship period of 10 cases with supervision of an expert robotic surgeon, called "proctor."

In addition, considering that indications for robotics in pediatric age are very limited and pediatric surgeons usually perform two to five robotic procedures per month, the presence of a robotic technician in the operative room may be useful for the first 6-12 months of the robotic activity to help in case of problems with the robot's setting. All these measures allowed to avoid the risk of intraoperative complications. In addition, most postoperative complications that occurred in our series were Clavien I-II grade whereas Clavien IIIb complications were reported in only $1.9 \%$ of our cases.

Our study confirmed that RAS has still a limited diffusion in Italy for pediatric patients. Before starting robotic activity, pediatric surgeons have to obtain a certificate by passing an exam after a virtual and experimental training period. A mentorship clinical period of 10 cases under supervision of a proctor is also needed. The main indications for RAS in children remain reconstructive urological procedures. RAS is safe in pediatric patients but its applications are currently limited to children older than 2 years and with a body weight $>15 \mathrm{~kg}$, due to the size of robotic ports.

\section{Disclosure Statement}

No competing financial interests exist.

\section{Funding Information}

No funding was received for this article.

\section{References}

1. Santoro E, Pansadoro V. Robotic surgery in Italy national survey. Updates Surg 2013;65:1-9.

2. Corcione F, Esposito C, Cuccurullo D, Settembre A, Miranda N, Amato F, Pirozzi F, Caiazzo P. Advantages and limits of robot-assisted laparoscopic surgery: Preliminary experience. Surg Endosc 2005;19:117-119.

3. Chen CJ, Peters CA. Robotic assisted surgery in pediatric urology: Current status and future directions. Front Pediatr 2019;7:90.

4. Howe A, Kozel Z, Palmer L. Robotic surgery in pediatric urology. Asian J Urol 2017;4:55-67.

5. Varda BK, Wang Y, Chung BI, et al. Has the robot caught up? National trends in utilization, perioperative outcomes, and cost for open, laparoscopic, and robotic pediatric pyeloplasty in the United States from 2003 to 2015. J Pediatr Urol 2018;14:336.e1-e336.e8.

6. Peters CA. Robotically assisted surgery in pediatric urology. Urol Clin North Am 2004;31:743-752.

7. Passerotti C, Peters CA. Pediatric robotic-assisted laparoscopy: A description of the principle procedures. Scientific World J 2006;6:2581-2588.

8. Spinoit AF, Nguyen H, Subramaniam R. Role of robotics in children: A brave new world! Eur Urol Focus 2017;3:172-180.

9. Andolfi C, Kumar R, Boysen WR, Gundeti MS. Current status of robotic surgery in pediatric urology. J Laparoendosc Adv Surg Tech A 2019;29:159-166.

10. Kim SJ, Barlog JS, Akhavan A. Robotic-assisted urologic surgery in infants: Positioning, trocar placement, and physiological considerations. Front Pediatr 2019;6:411.

11. Cundy TP, Harling L, Hughes-Hallett A, Mayer EK, Najmaldin AS, Athanasiou T, et al. Meta-analysis of robotassisted vs conventional laparoscopic and open pyeloplasty in children. BJU Int 2014;114:582-594. 
12. Avery DI, Herbst KW, Lendvay TS, Noh PH, Dangle P, Gundeti MS, et al. Robot-assisted laparoscopic pyeloplasty: Multi-institutional experience in infants. $J$ Pediatr Urol 2015;11:139. e1-e5.

13. Mizuno K, Kojima Y, Nishio H, Hoshi S, Sato Y, Hayashi Y. Robotic surgery in pediatric urology: Current status. Asian J Endosc Surg 2018;11:308-317.

14. Dindo D, Demartines N, Clavien PA. Classification of surgical complications. A new proposal with evaluation in a cohort of 6336 patients and results of a survey. Ann Surg 2004;240:205-213.

15. Jaffray B. Minimally invasive surgery. Arch Dis Child 2005;90:537-542.

16. Dakin GF, Gagner M. Comparison of laparoscopic skills performance between standard instruments and two surgical robotic systems. Surg Endosc 2003;17:574-579.

17. Hubens G, Coveliers H, Balliu L, Ruppert M, Vaneerdeweg W. A performance study comparing manual and robotically assisted laparoscopic surgery using the da Vinci system. Surg Endosc 2003;17:1595-1599.

18. Ramalingam M, Kallappan S, Nachimuthu S. A prospective comparative study of continuous and interrupted suturing in laparoscopic pyeloplasty in 3D era. J Laparoendosc Adv Surg Tech A 2018;28:1275-1278.

19. Gundeti MS, Kojima Y, Haga N, et al. Robotic-assisted laparoscopic reconstructive surgery in the lower urinary tract. Curr Urol Rep 2013;14:333-341.

20. Esposito C, Masieri L, Blanc T, Manzoni G, Silay S, Escolino M. Robot-assisted laparoscopic pyeloplasty (RALP) in children with horseshoe kidneys: Results of a multicentric study. World J Urol 2019;37:2257-2263.

21. Escolino M, Masieri L, Valla JS, Lopez PJ, Tokar B, Mushtaq I, Esposito C. Laparoscopic and robotic-assisted repair of retrocaval ureter in children: A multi-institutional comparative study with open repair. World J Urol 2019;37: 1941-1947.

22. Subramaniam R. Current use of and indications for robotassisted surgery in paediatric urology. Eur Urol Focus 2018;4:662-664.
23. Sodha S, Nazarian S, Adshead JM, Vasdev N, Mohan-S G. Effect of pneumoperitoneum on renal function and physiology in patients undergoing robotic renal surgery. Curr Urol 2016;9:1-4.

24. De Waal EEC, Kalkman CJ. Haemodynamic changes during low-pressure carbon dioxide pneumoperitoneum in young children. Pediatr Anesth 2003;13:18-25.

25. Chang C, Steinberg Z, Shah A, Gundeti MS. Patient positioning and port placement for robot-assisted surgery. J Endourol 2014;28:631-638.

26. Sorensen MD, Delostrinos C, Johnson MH, Grady RW, Lendvay TS. Comparison of the learning curve and outcomes of robotic assisted pediatric pyeloplasty. J Urol 2011;185(6 Suppl):2517-2522.

27. Sorensen MD, Johnson MH, Delostrinos C, Bice JB, Grady RW, Lendvay TS. Initiation of a pediatric robotic surgery program: Institutional challenges and realistic outcomes. Surg Endosc 2010;24:2803-2808.

28. Steyaert H, Van der Veken E, Joyeux L. Implementation of robotic surgery in a pediatric hospital: Lessons learned. J Laparoendosc Adv Surg Tech A 2019;29:136-140.

29. Andolfi C, Umanskiy K. Mastering robotic surgery: Where does the learning curve lead us? J Laparoendosc Adv Surg Tech A 2017;27:470-474.

30. Blavier A, Gaudissart Q, Cadiere GB, Nyssen AS. Comparison of learning curves and skill transfer between classical and robotic laparoscopy according to the viewing conditions: Implications for training. Am J Surg 2007;194: 115-121.

Address correspondence to:

Ciro Esposito, $M D$, PhD

Pediatric Surgery Unit

“Federico II" University of Naples

Via Pansini 5

Naples 80131

Italy

E-mail: ciroespo@unina.it 